\title{
Cuidados enfermeros en la España del siglo XVII: el manual de enfermería de Simón López
}

Nursing care during seventeenth century in Spain: Nursing manual belonging to Simon Lopez

\section{Cuidados de enfermagem no século XVII na Espanha: manual de enfermagem Simon Lopez}

Sonia Sánchez Aragó

Enfermera en Unidad de Cirugía del Hospital Nuestra Señora de Gracia, Máster en bioética y bioderecho.

Cómo citar este artículo en edición digital: Sánchez Aragó, S. (2016). Cuidados enfermeros en la España del siglo XVII: el manual de enfermería de Simón López. Cultura de los Cuidados (Edición digital), 20( 46).

Disponible en: < http://dx.doi.org/10.14198/cuid.2016.46.08>

Correspondencia: Calle Perpetuo Socorro 4,5A. CP.50006. Zaragoza.

Correo electrónico: sonits@@hotmail.com

Recibido: 08/02/2016; Aceptado: 22/08/2016

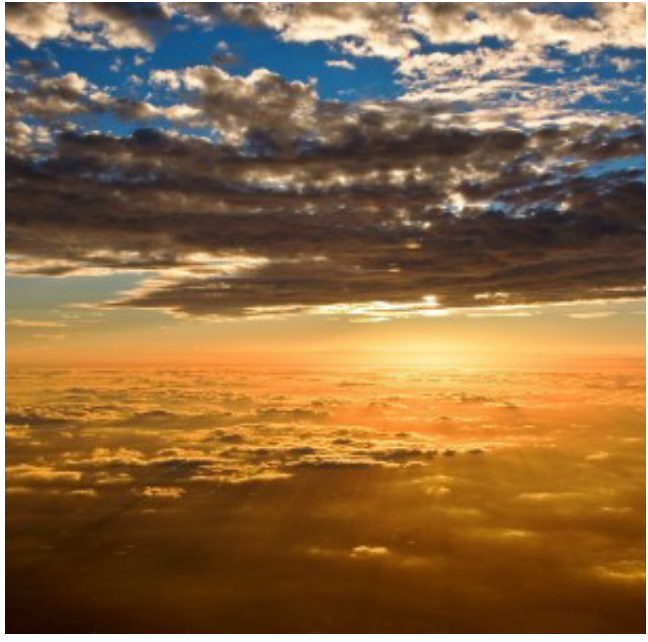

\section{ABSTRACT}

Objective: Throughout the different historical periods, care has been studied nursing. Speaking of the history of Spanish Nursing, it is generally accepted by most researchers as a key date institutionalization and professionalization of the last century, and more specifically that of 1857 . In this case, it is intended to expose the origins of a trade, as was the nurse or care was exercised in hospitals in the seventeenth century in Spain, see how were the tasks and roles played these caregivers, recognition and social acceptance of trade among others.

Methods: To carry out this work has used the work Directory Nurses, whose full title is Direct Nurses and architect of charity to cure diseases of the body.

Results: For its purpose and content, the work Directory Nurses, Simon Lopez, is a key to know the Spanish Nursing seventeenth century.

Conclusions: The work offers a comprehensive view of the person taking into account the biological , psychological and social sphere, along with the human aspect of care.

Keywords: history of nursing, cares, nursing, health.

\section{RESUMO}

Objectivo: Ao longo dos vários períodos históricos, o cuidado tem sido estudado enfermagem. Falando da história da Enfermagem espanhola, é geralmente aceite pela maioria dos pesquisadores como uma institucionalização chave de data e profissionalização do século passado, e mais especificamente a de 1857. 
Neste caso, pretende-se expor o origens de um comércio, como era a enfermeira ou o cuidado foi exercido em hospitais no século XVII na Espanha, ver como eram as tarefas e papéis desempenhados esses cuidadores, o reconhecimento ea aceitação social do comércio, entre outros.

Metodologia: Para realizar este trabalho utilizou os enfermeiros Diretório de trabalho, cujo título completo é Nurses directos e arquiteto da caridade para curar doenças do corpo

Resultados: Para seu propósito e conteúdo, as enfermeiras Diretório trabalho, Simon Lopez, é a chave para conhecer a Enfermagem do século XVII espanhol.

Conclusão: $O$ trabalho oferece uma visão abrangente da pessoa tendo em conta a esfera biológica, psicológica e social, juntamente com o lado humano de cuidados.

Palavras-chave: história da enfermagem , cuidado, nutrição, saúde.

\section{RESUMEN}

Objetivo: A lo largo de las distintas épocas históricas, el cuidado ha sido objeto de estudio de la enfermería. Al hablar de la historia de la Enfermería española, se suele aceptar por la mayoría de los investigadores como fecha clave de su institucionalización y profesionalización la de mediados del siglo pasado, y más concretamente la del año 1857. En este caso, se pretende exponer los orígenes de un oficio, como era el de enfermero o cuidados que se ejercía en los hospitales en el siglo XVII en España, ver cómo eran las tareas y funciones que desempeñaban estos cuidadores, el reconocimiento y aceptación social del oficio entre otros.

Metodología: Para llevar a cabo el presente trabajo se ha utilizado la obra Directorio de Enfermeros, cuyo título completo es Directo de Enfermeros y artífice de obras de caridad para curar las enfermedades del cuerpo.

Resultados: Por su finalidad y contenido, la obra Directorio de Enfermeros, de Simón López, es una pieza clave para conocer la Enfermería español del siglo XVII.

Conclusión: La obra ofrece una visión integral de la persona teniendo en cuenta la esfera biológica, psicológica y social, junto con la vertiente humana de los cuidados.

Palabras clave: historia de enfermería, cuidados, enfermería, salud.

\section{INTRODUCCIÓN}

A lo largo de las distintas épocas históricas, el cuidado ha sido objeto de estudio de la enfermería, ha evolucionado a través del saber filosófico, los avances científicos y el desarrollo, dando lugar a un cambio en los roles de nuestra disciplina (Siles González,2004).

Al hablar de la historia de la Enfermería española, se suele aceptar por la mayoría de los investigadores como fecha clave de su institucionalización y profesionalización la de mediados del siglo pasado, y más concretamente la del año 1857.Es en ese año cuando se promulgó la Ley de Bases para la Instrucción Pública, conocida como Ley Moyano por el nombre del ministro que la auspició. Esta ley estableció la creación de los denominados Practicantes, que agrupaban a los anteriores, más Callistas, Dentistas (hasta 1877) y asistentes a partos (Gonzalez,2000).

A medida que nos remontamos en el tiempo, las complicaciones son mayores, debido fundamentalmente, a la escasez de documentación generada y conservada hasta nuestros días (García Martínez,1998). Desde comienzos del siglo XVI, los hospitales españoles no dejaron de evolucionar, intentando incorpo- 
rar, en la medida de sus posibilidades, las nuevas técnicas y conocimientos que el desarrollo científico de la época proporcionaba (García Martínez, 2004).

El siglo XVII fue testigo de los primero intentos de explicar la mecánica del cuerpo Humano (Hernández Martín,1996). Jerome Fabricus 1619 fue el primer embriólogo el desarrollo de método clínico fue contribución de Thomas Sydeham 1624, propuso nuevos métodos en el cuidado de los enfermos, es el propulsor de sustituir el ambiente cargado de la habitación por aire fresco, se descubrió también la utilidad de la autopsia. (Martínez, 2004)

Durante los siglos XVII y XVIII causaron estragos, hubo brotes devastadores e tifus, peste bubónica, el estilo de vida urbano empezaba a favorecer estas enfermedades, la higiene era deficiente, la carencia de instalaciones sanitarias, la depuración de las aguas residuales era inadecuada y no había leyes de salud pública (López, 2001).Todos los acontecimientos acaecidos en las diversas épocas han forjado la historia de la profesión enfermera (Hernández Martín, 1997).

\section{MÉTODO}

Para poder adentrarse en la esfera del conocimiento del pasado de cualquier fenómeno son cada vez más utilizados los métodos etnohistóricos (Hernández Cortina,2004).En este caso, se pretende exponer los orígenes de un oficio, como era el de enfermero o cuidados que se ejercía en los hospitales en el siglo XVII en España, ver cómo eran las tareas y funciones que desempeñaban estos cuidadores, el reconocimiento y aceptación social del oficio entre otros (Burgos Moreno, 2009).

Para llevar a cabo el presente trabajo se ha utilizado la obra Directorio de Enfermeros, cuyo título completo es Directo de Enfermeros y artífice de obras de caridad para curar las enfermedades del cuerpo (Hernández Cortina, 2004).

Los datos sobre el autor de la obra son escasos. Por las referencias que se han encontrado, es una persona castellana de origen, enfermero y barbero de profesión que trabajó ejerciendo la enfermería durante más de dos décadas en distintos hospitales castellanos (Burgos Moreno,2009). Se deduce que es un hombre culto, $y$ que dominaba los clásicos en latín y castellano y los principales autores de su época; ya que en su única obra cita a unos cincuenta (GarcíaCarpintero Blas, 2007).

El primer borrador se fechó en 1651 y en cuanto a la fecha exacta de su terminación, en sus páginas prologales figura la de 1668. Se divide en ocho tratados y 150 capítulos, consta de un total de 565 páginas de texto (junto con otras 22 de índices y aprobaciones) y seguramente quedó manuscrita a causa de la censura (López, 1668).

Los títulos de los tratados son los siguientes: El Tratado 1 (capítulos 1 a 9) se titula: "De las casos contingentes, en el qual se incluien todos quantos géneros de unturas ay y se pueden hacer en todo género de enfermedades con el Méthodo que combiene, según el buen orden de la Medicina”. En segundo lugar, el Tratado 2 (capítulos 20 a 33) titulado "De otros casos contingentes en muchas enfermedades menudas, con algunos remedios en los principios de ellas". En tercer lugar, el Tratado 3 (capítulos 34 a 40) se titula: "De cómo se han de dar los xaraues $y$ asistir a las sangrías y hechar los clisteres $y$ supositorios o calas". Por otro lado, el Tratado 4 (capítulos 41 a 56) se titula "De las fluxiones de vientre con las disenterías propias y impropias". En quinto lugar, el Tratado 5 (capítulos 57 a 108) se titula "De las fiebres agudas y de las ter- 
cianas y quartanas, con el regimiento de ellas, y cómo nos emos de regir en tiempo de peste y preuenciones para ella”. En sexto lugar, el Tratado 6 (capítulos 109 a 137) se titula "De otras enfermedades, assí crónicas como ereditarias y accidentales". En penúltimo lugar, el Tratado 7 (capítulos 138 a 148) se titula "De la calidad del agua y quál es la mexor, y de todo género de aguas cogidas que beuen los enfermos y el modo de cogerlas con su peso y medida". Por último el Tratado 8 comprende los capítulos 149 y 150 y se titula "De la calidad de los mantenimientos simples que nos sirben para nuestra conseruación" (López, 1668).

Además, lleva incorporada tres aprobaciones, todas ellas de insignes médicos de la época, como la de D. Juan Lázaro Gutiérrez (catedrático de propiedad de Medicina en la Real Universidad de Valladolid), el D. Geromo Pardo (catedrático de Methodo Medendi en la Real Universidad de Valladolidad) y el Dr. Juan de Río Noriega, médico de la ciudad de Rioseco y catedrático en de la Universidad de Salamanca (López,1668).

Esta obra constituye un precedente en los textos modernos de enfermería, que junto al manual realizado por la Mínima Congregación de los Hermanos Enfermeros Pobres titulado: Instrucción de enfermeros, para aplicar los remedios a todo género de enfermedades, $\mathrm{y}$ acudir a muchos accidentes que sobrevienen en ausencia de los Médicos, aunque más conocido como "enfermeros obregones", y en su segunda edición (1625), ampliada por el Hermano Andrés Fernández, constituyen las obras clásicas sobre la enfermería del siglo XVII español (López, 1668).

\section{DESARROLLO}

La práctica de la enfermería abarca tanto el cuidado de las personas sanas como las han de- sarrollado un problema de salud, así como las que se encuentran en peligro de desarrollarlo. Enfermería juega un papel fundamental en el fomento de la salud así como en la prevención. La obra ha sido concebida, fundamentalmente, para la formación del enfermero, tanto el que se iniciaba en el oficio como el que ejercía la profesión. Ya en la obra Directorio de enfermeros encontramos descritas actividades encaminadas a cubrir las necesidades básicas del ser humano. La función asistencial es la más desarrollada en la obra de las funciones que se llevaban a cabo por parte de enfermería, aunque también se halla plenamente desarrollada a lo largo del material la actividad docente, con el fin de proporcionar a los enfermeros los conocimientos teóricos y prácticos imprescindibles para el ejercicio de la profesión (García Martinez,1993).

Virginia A. Henderson definía la profesión enfermera, con las siguientes palabras: «Creo que la enfermera es, y debe ser legalmente, un profesional independiente capaz de hacer juicios independientes mientras no haga diagnósticos, prescriba tratamientos o emita pronósticos, porque éstas son las funciones del médico. Pero la enfermera es la mayor autoridad en los cuidados básicos de enfermería. Quizá deba explicar que por cuidados básicos de enfermería me refiero a ayudar a los pacientes en las siguientes actividades o darles los conocimientos necesarios para que puedan llevarlas a cabo sin ayuda [se nombran 14 actividades]» (García Martínez,1993). En la obra de Simón López, escrita tres siglos antes, se tiene interés en formar al enfermero, pero también al paciente, con la finalidad de que este se valiese por sí mismo existiendo una relación con la definición de la autora Virginia Henderson. Por ello, se han agrupado las distintas necesidades del ser humano en grupos con el 
fin de comprender las actividades, tareas y técnicas enfermeras que realizaba el enfermero de la época (López, 1668).

En lo que respecta a la respiración, se describe patología respiratoria tales como asma, pleuresía, taudardillo entre otras. Para tratar las dolencias eran muy empleados los sahumerios que consistía en producir humo de una sustancia aromática que se quema y poseía una finalidad broncodilatadora y expectorante (Siles González, 2007). También se hablaba del ambiente que debía proporcionar el enfermo, recogido en los manuales de hoy en día. Así en la obra de Simón López se expresaba: "El aposento para estos enfermos de dolor de costado ha de ser abrigado; mayormente en imbierno hanse de bestir las paredes o, por lo menos, la alcoba, si la hubiere, con algunos paramentos $y$, el suelo, con esteras. Siempre ha de hauer un brasero de lumbre de brasa de leña o de carbón muy bien ençendido (porque el tufo tapa la respiración)" (López, 1668).

La alimentación y dietética ocupan un lugar primordial en la formación del enfermero. En la obra se expone la importancia de los alimentos en la mejora del proceso del enfermedad, para ello, se establecen las características de los alimentos y se insiste en la creación de hábitos saludables. Por ejemplo, se explica a los enfermeros la forma de fabricar un embudo de latón y se da importancia que el paciente mantenga una postura adecuada al darle de comer. El autor explica a los enfermeros, de forma clara y detallada, la forma de fabricar el embudo de latón y la postura del paciente para darle de comer: "El tamaño del embudo será como seys o siete dedos de trauessía con copa y todo y de largo; el cañón de la copa para abajo no ha de ser derecho, sino algo arqueado, al modo de una cornucopia; la punta deste embudo no ha de quedar redonda sino balada,

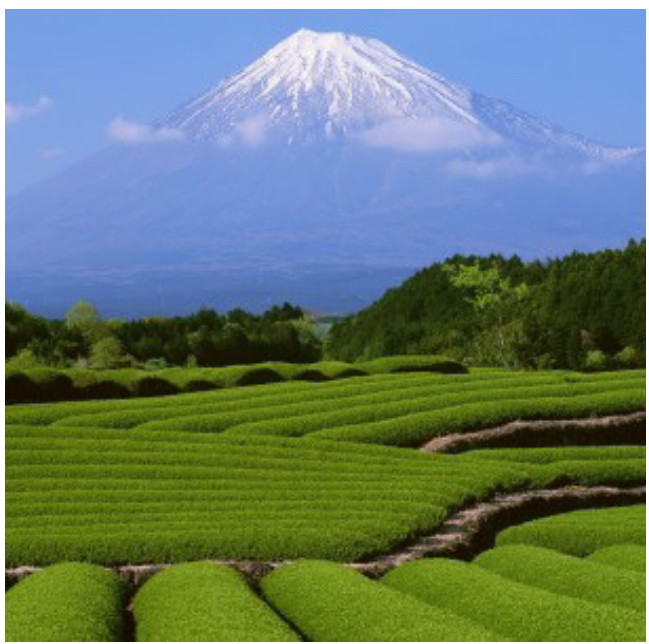

porque con esto no abre la boca tanto y puede mejor paladear." (García Martínez, 1993).

Con lo que respecta al patrón de eliminación, Simón López propone una serie de medidas consistentes en la administración de enemas, calas o clisteres para esos casos. En el texto se nombra la estangurria que consiste en la dificultad para orinar (López,1668). Para ello, se formulan diversas formas de actuar, como la ingesta de bellota por sus propiedades diuréticas, así como la toma de hojas de rábano cocidas en caldo de puchero. Los capítulos 37,38 y 39 recogen la composición de los clisteres y la forma de administrarlos (Siles González, 2007).

La necesidad de sueño y descanso también se encuentra en esta obra. El papel del enfermero se encaminaba hacia la vigilancia, a conseguir un ambiente óptimo que facilitase el sueño. También se indica cómo administrar medicación hipnótica e inductora del sueño, preparada fundamentalmente a base de plantas como por ejemplo, la papaver somniferum. El capítulo 68 está completamente dedicado al descanso de los enfermos frenéticos. También el empleo de musicoterapia, la creación de un ambiente relajado y la presencia de flores es- 
taban ya presentes en esta obra del siglo XVII (García Martínez, 1993).

Con respecto a la necesidad de termorregulación encontramos medidas encaminadas a mantener la temperatura corporal. En el capítulo 28 titulado "del sudor, quál es bueno o malo y cómo se conocerá y qual es el que deue guardar y quál no" se expresa: "Muchas ocasiones se le ofreçerán al enfermero con enfermos de calenturas malignas donde suele aber sudor, en los días judiçiarios, y porque tenga alguna notiçia quando les biniere estos sudores para abisar al Médico y bea lo que se ha de haçer" (López, 1668).

En la necesidad de higiene, se recalca en la obra, que su carencia o deficiencia provocan y favorecen la propagación de las enfermedades. Por ello, entre las actividades que debe realizar los enfermeros se destaca bañar al paciente, cambiar la ropa y mantener limpios los aposentos. Además se recalca en la importancia de aplicar medidas de higiene y desinfección durante las epidemias, así como el lavado de manos (López, 1668).

En estrecha relación, se encuentra la seguridad física, ya que puede poner en peligro la integridad del enfermo si no se tiene en consideración. En el capítulo 66 se recalcan las medidas de seguridad que deben ser empleadas por el enfermero ante un enfermo delirante (Siles González,2007). Así se expresa en la obra: "Han suçedido y suçeden oy tantas desgracias y fatales fines con enfermos frenéticos, por no cuidar con diligengia y atençión a su asistençia, que fuera nunca acabar el aberlos de referir. $Y$, no hay duda, de que si preguntaran en cada comunidad o hospital algunos casos lastimosos de frenéticos, que todos tendrían arto que deçir de enfermos que se desgraçiaron por falta de asistençia y ignorançia de los enfermeros o assistentes" (López, 1668).
También se hace hincapié en la obra de las necesidades psicológicas y espirituales. En la obra se considera la esfera biológica, espiritual y social de la persona. Por ello, el enfermero debe hablar con el enfermo, alegrarle y animarle, ponerle música y en definitiva ganarse la confianza del enfermo (López, 1668).

Por otro lado, la obra también apuesta por enseñar al enfermo, es decir, por el autocuidado. En la página 216 de la obra se muestra este aspecto: "Y si esto puede hacer el enfermo, le enseñará el enfermero cómo lo ha de hacer, teniendo cuidado de renouarle a menudo las dos escudillas o basos de agua fría...”. En otras páginas también se expresa este concepto de autocuidado, así en la página 267: “"... y enseñar al enfermo cómo ha de tomar los lamedores $y$ cómo ha de acer los gargarismos y otras cosas que aora se dirán que le tocan, de todo lo qual ya dexo dicho arto en sus lugares donde podrá recurrir, por no me detener aquí más»" (López, 1668).

Por último, también se encuentran en la obra consejos y recomendaciones dirigidos a la comunidad con respecto a la adopción de medidas higiénicas y medioambientales (López, 1668). En siglo XVII las epidemias estaban muy presentes, por lo tanto también se expresa en la obra medidas para evitar la propagación de enfermedades infecciosas, entre ellas destaca la purificación del aire, cubrir con cal los cadáveres (García Martínez, 2004). La obra establece un amplia relación de enfermedades $\mathrm{y}$ afecciones, medidas preventivas y curativas, que nos proporcionan una visión de este oficio en este siglo. (García Martínez, 1993)

\section{CONCLUSIONES}

De todos los estudios realizados sobre los hospitales fundados entre fines de la Edad Media y toda la Edad Moderna, los dedicados a 
los cuidados dispensados a los enfermos durante su estancia en estos centros y al personal sanitario que los proporcionaba, las enfermeras y enfermeros, han sido escasos, aun sabiendo que la esencia de estas instituciones y su razón de ser fundamental es precisamente ésa: proporcionar cuidados de salud y curar (Siles González, 2000).

Por su finalidad y contenido, consideramos a Directorio de Enfermeros, de Simón López, una obra clave para conocer la Enfermería español del siglo XVII (García Martínez,1993).

En la obra de Simón López, se aprecia un interés por delimitar el trabajo de los profesionales que ejercen en el hospital en esa época (barbero, boticario, médico) insistiendo sobre todo en la labor de enfermería (López, 2001). Por eso, ya se encuentran reflejadas las cuatro funciones que en la actualidad se le reconoce a la profesión enfermera: docencia, asistencia, investigación y administración, siendo las dos primeras, con gran diferencia, las más ampliamente desarrolladas (Hernández Martín, 1997).

A lo largo de la obra, se intenta explicar las técnicas y las tareas llevadas a cabo por enfermería de forma razonada y haciendo fácil su compresión mediante la utilización de diversos recursos didácticos tales como comparaciones y ejemplos (Hernández Martín, 1996).

El trabajo del enfermero de la época queda expuesto a lo largo de la obra de Simón López, especialmente en el prólogo, en las que destacan las siguientes funciones y tareas: ejecutar las prescripciones ordenadas por el médico, proveer lo necesario a la hora de realizar curas, mantener unas condiciones óptimas de higiene y limpieza, asistir al enfermo en ausencia del médico o en situaciones de urgencia, observar la evolución del enfermo e informar al médico de los acontecimientos, dar de comer al enfermero en las condiciones adecuadas y administrar medicación pauta explícitamente por el médico (Hernández Martín, 1997). Así se ha encontrado una correlación entre la definición de enfermería de Virgina Henderson y las necesidades básicas expresadas por ésta, con los contenido de la obra expuestos tres siglos antes. En definitiva, la obra ofrece una visión integral de la persona teniendo en cuenta la esfera biológica, psicológica y social, junto con la vertiente humana de los cuidados (Hernández Cortina, 2004).

\section{BIBLIOGRAFÍA}

- Burgos Moreno, M., y Paravic Klijn, T. (2009). Enfermería como profesión. Revista cubana de enfermería, 25(1-2). Disponible en: http:// scielo.sld.cu/scielo.php?script=sci arttext\&pid $=$ S0864-03192009000100010. Fecha de acceso 21 de abril de 2016.

- García-Carpintero Blas, E. (2007). Reflexión del papel de la enfermería a lo largo de la historia. Enfermería Global, 6(2),1-6.

- García Martínez A.C., García Martínez M.J., Hernández Martín F., Pérez Melero A. y Pinar García M.E. (1993). Aproximación a la Enfermería española de los siglos XVIXVII. Presentación y análisis de la obra Instrucción de enfermeros, de Andrés Fernández, 1625. Madrid: Edición a cargo del Consejo General de Colegios de Diplomados en Enfermería.110-232.

- García Martínez, M.J., y García Martínez, A.C.(1998). La enseñanza de la Enfermería en la España del siglo XVII. Cultura de los Cuidados, 2(3),15-23.

- García Martínez, M.J.( 2004). Cuidados enfermeros en la España del siglo XVII. Hacia la búsqueda de una identidad profesional. Gazeta de Antropología, 20, 22.

- García Martínez, M. J. (2004). El método en la enfermería: una mirada desde la historia (siglo XVII). Metas de enfermería, 7(10), 58-64.

- Hernández Martín, F.J. (1996). Historia de la Enfermería en España (desde la Antigüedad hasta nuestros días). Madrid: Editorial Síntesis. 
- Hernández Martín, F., Gallego Lastra, R. D., Alcaraz González, S., González Ruiz, J. M. (1997). La enfermería en la historia. Un análisis desde la perspectiva profesional. Cultura de los cuidados, 2(3), 21-35.

- Hernández Cortina, A., Guardado de la Paz, C. (2004). La enfermería como disciplina profesional holística. Revista cubana de enfermería, 20(2), 1-1.

- López, S. (1668). Directorio de enfermeros y artífice de obras de caridad para curar las enfermedades del cuerpo Signatura:Manuscrito Ms 259. Biblioteca Universitaria de Salamanca.

- López, S., García Martínez, A. C. y García Martínez, M.
J. (2001). Directorio de enfermeros y artifice de obras de caridad para curar las enfermedades del cuerpo. Madrid: Enfermundi.

- Siles González, J.(2004). La construcción social de la Historia de la Enfermería. Index de enfermería, 47(13),7-10.

- Siles González, J. (2000). Antropología, historia y enfermería. Cultura de los cuidados: Revista de enfermería y humanidades, 4(7-8),5-7.

- Siles González, J., Solano Ruiz, M. D. C. (2007).

El origen fenomenológico del "cuidado" y la importancia del concepto de tiempo en la historia de la enfermería. Cultura de los cuidados, 11(21), 19-27.

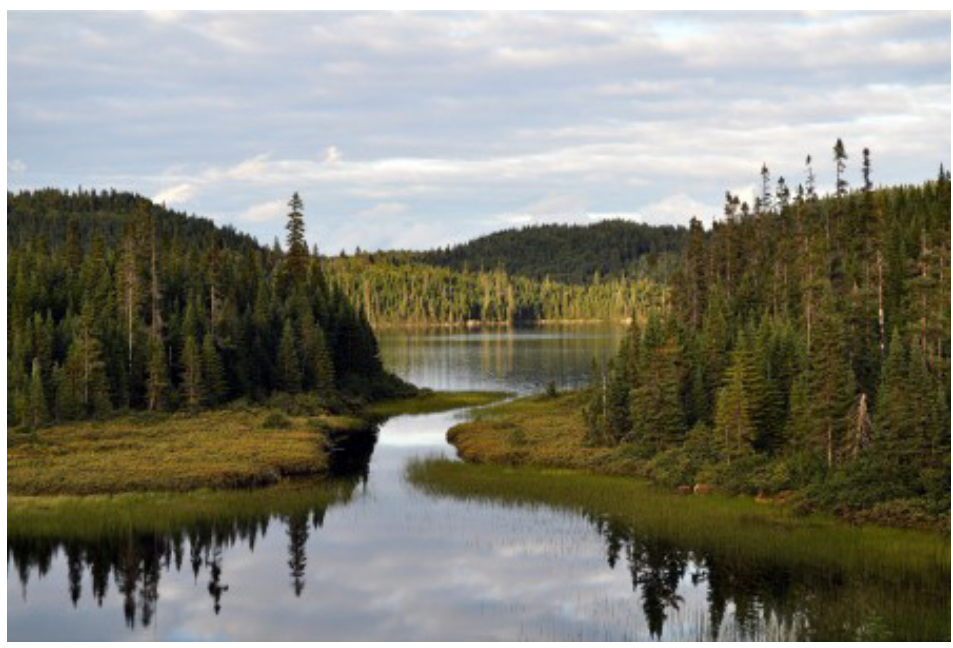

